\title{
Statistical Behavior and Symmetry Tests
}

\author{
G. E. Mitchell \\ North Carolina State University, Raleigh, NC 27695 \\ and Triangle Universities Nuclear Laboratory, Durham, NC 27708, USA \\ and J. F. Shriner, Jr. \\ Tennessee Technological University, Cookeville, TN 38505, USA
}

Received on 11 September, 2003

\begin{abstract}
Symmetries and statistical properties in nuclei are closely related. The most striking example is the extremely large enhancement of parity violation in neutron resonances. Statistical distributions can provide information about the underlying character of nuclear properties. Level statistics and electromagnetic transition distributions have been used successfully to provide unique tests of predictions of random matrix theory.
\end{abstract}

\section{Introduction}

The topic of fundamental symmetries is central to nuclear physics. The issue of whether the fundamental interactions are modified in the nuclear many-body system and how the physical observables are affected by the many-body environment is the subject of many investigations. Since at least under some circumstances the nucleus is well described by statistical approaches such as Random Matrix Theory (RMT), it seem appropriate to examine the interplay between symmetries and statistical concepts. In addition to fundamental symmetries, the nucleus displays a number of approximate symmetries (isospin, the $\mathrm{K}$ quantum number, $\mathrm{F}$ spin, etc.) which are crucially important to practicing nuclear physicists. The interplay between these approximate symmetries and statistical properties is also interesting.

We restrict the considerations here to discrete symmetries. This restriction is not essential, since there have been many proposed experiments that utilize proposed enhancements arising from stochastic properties. However, none of these proposed experiments have been performed [1-5].

For fundamental symmetries we consider parity as the classic example of the effect of statistical properties on physical observables. As we shall discuss below, the size of the measured longitudinal asymmetry (helicity dependence of the total cross section) for neutron resonances in heavy nuclei is amplified by a factor of $10^{6}$ relative to the longitudinal asymmetry measured in the nucleon-nucleon system. The details of these experimental results, as well as the historical background, are presented in a comprehensive review [6].

For an approximate symmetry we consider isospin. We have performed a series of measurements on the effect of isospin symmetry breaking on level statistics $[7,8]$ and on electromagnetic transitions $[9,10]$. The most striking feature is that a small symmetry breaking can have a large impact on the level statistics and on the transition distributions.
Work on the weak interaction in nuclei has always had a dual role. Understanding the effective weak interaction in nuclei is a basic problem of many-body theory. However, one can also use the weak interaction as a probe of this strongly interacting system. In a similar manner one can use statistical concepts (RMT) to learn about nuclear properties, but one can also use the nucleus as a laboratory to test explicit predictions of RMT.

\section{Parity violation in neutron reso- nances}

The strength of the weak interaction is about $10^{-7}$ that of the strong force. In nuclei the weak force has been studied through measurement of parity violating observables in nucleon-nucleon scattering, in few-body systems, and in light nuclei. The latter measurements (which require detailed knowledge of the wave functions involved) were summarized by Adelberger and Haxton [11].

A completely different approach to experiment and analysis involves neutron resonances in medium and heavy nuclei. For some two decades (approximately 1960 to 1980) there were sometimes conflicting reports about parity violation in neutron-induced reactions. The now accepted explanation for the very large enhancements that were sometimes observed was provided by Sushkov and Flambaum [12]. They estimated the size of the weak matrix elements $V_{s p}^{J}$ between $s$ - and $p$-wave resonances to be $\approx 1 \mathrm{meV}$ and predicted a longitudinal asymmetry

$$
p=\frac{\sigma_{+}-\sigma_{-}}{\sigma_{+}+\sigma_{-}}=2 \sum_{s} \frac{V_{s p}^{J}}{E_{s}-E_{p}} \sqrt{\Gamma_{n s} / \Gamma_{n p}}
$$

for the + and - helicity cross sections $\sigma_{+}$and $\sigma_{-}$for $p$-wave compound nuclear $(\mathrm{CN})$ resonances in specific nuclei. The predicted values were first observed at JINR, Dubna [13,14]. 
The qualitative explanation for the large asymmetries observed is that very large $s$-wave resonances are mixed into very small $p$-wave resonances, producing what is usually called kinematical enhancement. (Note that the kinematical enhancement is specific to the particular measurement of the helicity dependence of the cross section for $p$-wave neutron resonances at epithermal neutron energies.) The matrix elements between these very complicated states (for heavy nuclei there are of the order of $10^{5}$ components in the $\mathrm{CN}$ wave functions) and the close spacings $\left(E_{s}-E_{p}\right)$ lead to another enhancement - usually called dynamical or statistical enhancement. (Note that in contrast to the kinematical enhancement, the statistical enhancement is generic. It should - and does - occur in general in other strongly interacting quantum systems [15].) The history and development of this subfield is briefly reviewed by Mitchell et al. [6].

Unfortunately experimental limitations on the neutron flux prevented the Dubna group from extending their seminal measurements to higher energies. The TRIPLE collaboration was formed to extend these parity violation measurements to higher neutron energies, to more resonances, and to a wider range of targets. The details of the experimental efforts of this group are given in the previously cited review [6]. In favorable cases the parity violation is apparent by inspection in the raw data. This is illustrated in Fig. 1.

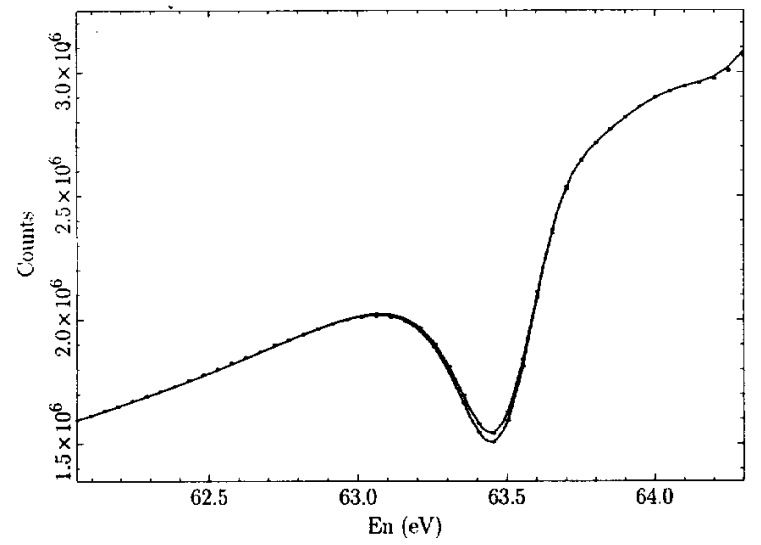

Figure $1 .{ }^{238} \mathrm{U}$ transmission spectra for two helicity states near the 63.4-eV resonance. The parity violation is apparent by inspection.

Although these very large longitudinal asymmetries (the largest measured value was 14\%), provided dramatic evidence for the essential correctness of the SushkovFlambaum theory, the general opinion was that these measurements were of only anecdotal value: since the wave functions were too complicated to be known, no quantitative information could be obtained.

The solution to this apparent dilemma was to adopt a purely statistical approach. The fact that the compound nucleus behaves statistically - Random Matrix Theory describes the fluctuation properties of the $\mathrm{CN}$ states - suggests that the weak matrix elements between the $\mathrm{CN}$ states be treated as random variables. The observed asymmetry - see
Eq. (1) - is a sum (over the contributions from the many $s$ wave states) of terms with the form of a product of the weak matrix element between the two states in question and of constants (spectroscopic parameters such as resonance energies and widths). Thus the measured asymmetry is the sum of random variables (the weak matrix elements between the $\mathrm{CN}$ states) and is itself a random variable. All of the weak matrix elements are sampled from the same distribution. Although it is not possible to determine the individual weak matrix elements, it is possible to determine from the set of measured asymmetries the variance of the distribution of these matrix elements. Thus the experimental result is the rms weak matrix element for a given nuclide. From these values it was possible to determine the effective weak nucleon-nucleus interaction. Within experimental errors this effective weak interaction is constant as a function of mass number.

Thus the stochastic nature of the highly excited nuclear system resulted in an enhancement of the physical observable (the longitudinal asymmetry) by a factor of about $10^{6}$ relative to the a priori expected value of $10^{-7}$. In addition, the statistical nature of the $\mathrm{CN}$ system led to a rather straightforward method of obtaining the rms weak matrix elements. The very complexity that was thought to preclude the determination of any detailed information was in fact the solution to the perceived problem. Although one of these enhancements (dynamical) is in fact generic and observed elsewhere, these parity violation neutron resonance experiments are the most striking example of the impact of the statistical nature of the system on a physical observable.

\section{Isospin violation}

\subsection{Effect of isospin symmetry breaking on level statistics}

Random Matrix Theory (RMT) has been employed in a wide variety of applications [16]. Although first introduced in the context of compound nuclear states by Wigner [17], there have been few experimental tests of RMT in nuclei. The primary reason is that the standard measures used to analyze the level statistics are sensitive to misassigned quantum numbers and to missing levels. These stringent requirements - usually called purity and completeness - have limited the experimental tests.

The role of symmetries in RMT is crucial. Since the symmetries govern the statistical distributions and there are many approximate symmetries in nuclei, it is natural to consider the effect of symmetry breaking on these distributions. The only direct way to test this is to find all of the states in an energy region and to determine all of their quantum numbers.

We chose to test RMT by measuring the effect of isospin symmetry breaking, since isospin is considered to be very well understood. To maximize the symmetry breaking we studied the nuclides ${ }^{26} \mathrm{Al}$ and ${ }^{30} \mathrm{P}$. These $N=Z=$ odd nuclides have the feature that the $T=0$ and $T=1$ states coexist from the ground state. 
The standard version of RMT that applies to nuclei is the Gaussian Orthogonal Ensemble (GOE). The GOE prediction for the distribution of the nearest neighbor spacings $S$ is very close to the Wigner distribution

$$
P_{G O E}(x)=\frac{\pi x}{2} \mathrm{e}^{-\pi \mathrm{x}^{2} / 4}
$$

where $x \equiv S / D$, with $D$ the average spacing. If isospin were a good quantum number, then the mixture of $T=0$ and $T=1$ states observed in the measurements should be a random mixture of two GOEs (or two Wigner distributions). Since isospin is known to be broken at the few percent level in these nuclei, one might expect only a small change from the two-GOE distribution. Experimentally the distribution is intermediate between the one-GOE and two-GOE distributions $[7,8,10]$. This agrees with predictions, first by Dyson [18] and later by Pandey [19] that a small symmetry breaking can have a large effect. The governing parameter is $\lambda=\alpha / D$, where $\alpha$ is the relative size of the symmetry breaking. This implies that even a very small symmetry breaking can have a large effect in a sufficiently dense spectrum.

A detailed analysis of the ${ }^{26} \mathrm{Al}$ data by Guhr and Weidenmüller [20] determined a Coulomb matrix element in agreement with values obtained in previous experiments. There are no other direct tests in nuclei, but there are extensive results in "analog" systems: acoustic resonances in quartz blocks [21] and electromagnetic resonances in in superconducting microwave billiards [22]. In both of these experiments the magnitude of the symmetry-breaking was varied - in the former case by adjusting the length of the transmission line between the billiards and in the latter case by physically removing part of the block. The results of these very good statistics experiments agree extremely well with RMT predictions.

\subsection{Effect of isospin symmetry breaking on electromagnetic transitions}

The GOE predicts that the amplitudes are Gaussian distributed. The observable is the square of the amplitude and the corresponding distribution is a $\chi^{2}$ of one degree of freedom, the Porter-Thomas (PT) distribution:

$$
P(y)=\frac{1}{\sqrt{2 \pi y}} e^{-\frac{y}{2}},
$$

where $y$ is the dimensionless strength parameter. For the study of electromagnetic transitions it is convenient to define $y=B(X L) /<B(X L)>$, where $\mathrm{B}$ is the reduced matrix element, $X$ is the character of the electromagnetic transition (electric or magnetic), and $L$ is the multipolarity of the transition. In addition the transitions are labeled by whether or not the (predominant) isospin changes. Because the different types of transitions have very different average strengths, the average matrix element must be determined separately for each $B(X L \Delta T)$. Since the reduced matrix elements vary over several orders of magnitude, it is convenient to rescale by changing to a new variable $z=\log _{10}(y)$.
The PT distribution becomes

$$
P(z)=\ln 10 \sqrt{y / 2 \pi} \exp (-y / 2) .
$$

The experimental procedures and the results for ${ }^{26} \mathrm{Al}$ and ${ }^{30} \mathrm{P}$ are presented in $[9,10]$. Although the strength of the conclusions is limited by the statistics, there are two rather striking results. The transition distributions do not agree with the PT distribution, and the distributions for the different types of transitions are not the same.

Although there were many data sets that agree with the PT distribution, there was no theoretical prediction for the effect of symmetry breaking on the distributions. Heuristic arguments strongly suggested that the transition distribution should not change from Porter-Thomas, in direct contradiction to the experimental results. However, the first theoretical studies $[23,24]$ did predict that the effect of symmetry breaking on the transition distributions is a change from the P-T distribution. There are no other experimental results in nuclei, but there is a recent result using coupled superconducting microwave billiards. Dembroski et al. [25] measured the effect of symmetry breaking on the strength distribution and observed a change from the PT distribution.

However, there is a new issue for the transition distributions. The effect of symmetry breaking on the eigenvalue distribution was a generic effect, which could be explained completely with RMT. The fact that RMT predicts a deviation from the Porter-Thomas distribution is certainly part of the story. However, if the distributions for E1 and M1 transitions (for example) are different, then this must be a dynamical effect which cannot be explained by a theory such as RMT. Thus the approach of using a high statistics "analog" measurement to supplement and confirm the experimental results cannot be used. In order to simulate the dynamics of the many-body problem, we are utilizing the shell model to study the effect of isospin symmetry breaking on the different types of electromagnetic transitions. Naturally this approach is somewhat model dependent (on the specific formulation to characterize the isospin symmetry breaking) and therefore not as straightforward as the RMT calculations. These analyses are now in progress [26]. The general conclusion for the electromagnetic transition is that contrary to conventional wisdom, symmetry breaking does change the transition distributions from the standard Porter-Thomas distribution. This opens the possibility to invert this logic and to use measured distributions of electromagnetic transitions to infer characteristics of the relevant approximate symmetries.

Thus the nucleus has proven to be a suitable laboratory in which to test predictions of statistical theory. In particular the prediction by Dyson [18] that a small symmetry breaking can have a large effect on the eigenvalue distribution was demonstrated explicitly for the first time. In addition, it was demonstrated for the first time that symmetry breaking does change the statistical distribution of electromagnetic transitions. These measurements preceded the theoretical analyses, which are consistent with the unexpected experimental results. 


\section{Summary and conclusion}

Symmetries and statistical properties are strongly connected in nuclei. Statistical distributions can provide information about the underlying character of nuclear properties. In favorable circumstances the statistical nature of the nucleus can lead to large enhancements of observables: the most striking example is the extremely large enhancement of parity violation in neutron resonances. The nucleus can also be used as a test laboratory. Level statistics and electromagnetic transition distributions have been used successfully to provide unique tests of predictions of random matrix theory.

\section{Acknowledgments}

This work was supported in part by the U.S. Department of Energy, Office of High Energy and Nuclear Physics, under grants No. DE-FG02-97-ER41042 and DE-FG0296ER40990.

\section{References}

[1] N. R. Roberson, C. R. Gould, and J. D. Bowman, eds., Tests of Time Reversal Invariance in Neutron Physics (World Scientific, Singapore, 1987).

[2] C. R. Gould, J. D. Bowman, and Y. P. Popov, eds., Time Reversal Invariance and Parity Violation in Neutron Reactions (World Scientific, Singapore, 1994).

[3] N. Auerbach and J. D. Bowman, eds., Parity and Time Reversal Invariance in Compound Nuclear States and Related Topics (World Scientific, Singapore, 1995).

[4] C. R. Gould, G. L. Greene, F. Plasil, and W. M. Snow, eds., Fundamental Physics with Pulsed Neutron Beams (World Scientific, Singapore, 2001).

[5] P. E. Koehler, C. R. Gould, R. C. Haight, and T. E. Valentine, eds., Astrophysics, Symmetries, and Applied Physics at Spallation Neutron Scources (World Scientific, Singapore, 2002).

[6] G. E. Mitchell, J. Bowman, S. I. Penttilä, and E. I. Sharapov, Phys. Rep. 354, 157 (2001).

[7] G. E. Mitchell, E. G. Bilpuch, P. M. Endt, and J. F. Shriner, Jr., Phys. Rev. Lett. 61, 1473 (1988).

[8] J. F. Shriner,Jr., E. G. Bilpuch, P. M. Endt, and G. E. Mitchell, Z. Phys. A 335, 393 (1990).
[9] A. A. Adams, G. E. Mitchell, and J. F. Shriner, Jr., Phys. Lett. B 422, 13 (1998).

[10] J. F. Shriner, Jr., C. A. Grossmann, and G. E. Mitchell, Phys. Rev. C 62, 054305 (2000).

[11] E. G. Adelberger and W. C. Haxton, Annu. Rev. Nucl. Part. Sci. 35, 501 (1985)

[12] O. P. Sushkov and V. V. Flambaum, JETP Lett. 32, 352 (1980).

[13] V. P. Alfimenkov, S. B. Borzakov, V. V. Thuan, Y. D. Mareev, L. B. Pikelner, D. Rubin, A. S. Khrykin, and E. I. Sharapov, JETP Lett. 34, 295 (1981).

[14] V. P. Alfimenkov, S. B. Borzakov, V. V. Thuan, Y. D. Mareev, L. B. Pikelner, A. S. Khrykin, and E. I. Sharapov, Nucl. Phys. A 398, 93 (1983).

[15] G. E. Mitchell, J. D. Bowman, and H. A. Weidenmüller, Rev. Mod. Phys. 71, 445 (1999).

[16] T. Guhr, A. Müller-Groeling, and H. Weidenmüller, Phys. Rep. 299, 189 (1998).

[17] E. P. Wigner, in Statistical Theories of Spectra: Fluctuations, edited by C. E. Porter (Academic Press, New York, 1965), p.199.

[18] F. J. Dyson, J. Math. Phys. 3, 1191 (1962).

[19] A. Pandey, Ann. Phys. (N.Y.) 134, 110 (1981).

[20] T. Guhr and H. A. Weidenmüller, Ann. Phys. (N.Y.) 199, 412 (1990).

[21] C. Ellegaard, T. Guhr, K. Lindemann, J. Nygård, and M. Oxborrow, Phys. Rev. Lett. 77, 4918 (1996).

[22] H. Alt, C. I. Barbosa, H.-D. Gräf, T. Guhr, H. L. Harney, R. Hofferbert, H. Rehfeld, and A. Richter, Phys. Rev. Lett. 81, 4847 (1998).

[23] C. I. Barbosa, T. Guhr, and H. L. Harney, Phys. Rev. E 62, 1936 (2000).

[24] M. S. Hussein and M. P. Pato, Phys. Rev. Lett. 84, 3873 (2000).

[25] C. Dembowski, Ph.D. thesis, Technische Universität Darmstadt (2003).

[26] J. F. Shriner, Jr., G. E. Mitchell, and B. A. Brown, unpublished. 